\title{
Optimal structure of groups under exposure to fake news
}

\author{
Evelin Berekméri ${ }^{1}$, Imre Derényi ${ }^{1,2}$ (D) and Anna Zafeiris ${ }^{1,2^{*}}$ (D)
}

\author{
*Correspondence: \\ lanna@hal.elte.hu \\ 'Department of Biological Physics, \\ Eötvös University, Pázmány P. stny \\ 1/A, 1117 Budapest, Hungary \\ ${ }^{2}$ MTA-ELTE Statistical and Biological \\ Physics Research Group, Pázmány \\ Péter stny. 1/A, 1117 Budapest, \\ Hungary
}

\begin{abstract}
Humans predominantly form their beliefs based on communication with other humans rather than direct observations, even on matters of facts, such as the shape of the globe or the effects of child vaccinations. Despite the fact that this is a well-known (not to say: trivial) observation, literature on opinion dynamics and opinion formation largely overlooks this circumstance. In the present paper we study the effects of limited access to information on the level of knowledge of members of groups embedded into an environment that can be observed. We also study the consequences of false information circulating within the group. We find that exposure to fake news makes intense communication counterproductive, but, at the same time, calls forth diversification of agents with respect to their information spreading abilities.
\end{abstract}

Keywords: Communication network, Optimal group structure, Limited access to information, Fake news, Disinformation, Diversity

\section{Introduction}

Humans believe in great many things. We have beliefs regarding history, art, proper and improper behaviour, law-systems, companies, climate change, child vaccination, etc. Many of these things are "social constructions" in the sense that they do not correspond directly to some kind of external reality, rather to some kind of shared abstract idea (for example 'proper behaviour' or 'law-system', from the above list) (Searle 2011). On the other hand, many of our ideas do refer to some kind of external reality (like climate change, the usefulness or harmfulness of child vaccination or the shape of the globe), which concepts are in intricate relations with the former ones (Berger and Luckmann 1991; Hacking 2000). What is common in all beliefs is that humans form them mostly based on communication with fellow humans rather than direct observations (Sloman and Fernbach 2017). This is a fundamental feature of all social constructions, but it firmly holds for scientific ideas as well: most of us have never measured or looked up statistical data regarding the effects of homeopathic medicament or the speed with which the polar ice layer attenuates, yet most of us have a clean-cut opinion in these matters.

The above examples refer to complex scientific problems in which the ratio of the data to which an average individual has access to is extremely small: observation data related to the climate change for example is far more abundant than being accessible for one person. Moreover, with the development of information technology, people are exposed to an ever increasing amount of data. By now, for an ordinary person under ordinary

(c) The Author(s). 2019 Open Access This article is distributed under the terms of the Creative Commons Attribution 4.0 International License (http://creativecommons.org/licenses/by/4.0/), which permits unrestricted use, distribution, and reproduction in any medium, provided you give appropriate credit to the original author(s) and the source, provide a link to the Creative Commons license, and indicate if changes were made. 
conditions, it is basically impossible to check the source and dependability of every piece of the received information, partly due to inefficient access to the source of information, partly simply due to lack of time - not to mention other, psychological and social factors (O'Connor and Weatherall 2018; Scheufele and Krause 2019). On the other hand, not all factual questions are this complex, as many of them can be decided based on only a few aspects - these cause debate more rarely.

In the present paper we focus on the effect of limited information access, in groups aiming to achieve a clear idea regarding an "external reality" which can be observed. We study the optimal structure of such groups - described by the communication network and the observation/communication activities - under various exposure levels to fake news. This model refers more to scientific fake news rather than political ones since in the present model we assume that there exists an observable external reality. Agents placed into a randomly generated, but observable environment can modify their beliefs either by direct observation (which is a costly activity) or via communicating with their fellow group members (which is a much less costly action). Agents are exposed to fake news during the entire run. The features of the most effective groups are then determined by optimizing the communication network and the activity levels (both communication and observation) for each agent, with a genetic algorithm. The fitness function is defined by the accuracy of the group after the run, minus the costs of the activities.

Although in the literature false information, fake news, misinformation and disinformation mean different things (disinformation contains intentionally distorted data while in case of misinformation the distortion can be unintended), in the present article we use them as synonyms, since from the point of view of our model, the reason why the information is false does not matter. We adjust the information-accessing abilities of the agents by a parameter called $H$ which refers to the portion of the environment an agent is able to observe (see also Fig. 1). Accordingly, it can be interpreted in two ways: the ability of the agents, assuming an environment with fixed complexity, or alternatively, the complexity of the environment, assuming that humans have (by and large) similar abilities (for example a simple "environment" can be comprehended by each agent individually, while if they are embedded into a more complex environment, then only a portion of it will be accessible).

Despite the fact that the limited nature of information access is a fundamental property of opinion dynamics and opinion formation, it has not gained attention up to now. In contrast, phenomena related to fake news have gained more and more attention recently.

Fake news exists since humans do, but its spread has always been subject to the current technologies of information transfer. In recent times, along with the development of communication technology, newer and newer channels of information distribution appeared which in the meantime led to initiations aiming to preserve the solidity and verity of news - such as the fairness doctrine introduced in the US in the middle of the 20th century (Iyengar and Massey 2018). However, the appearance of internet and not much later the break-in of social media made the publishing and the dissemination of news cheaper and easier, thus it has become the most important platform of false news, too. Unreal news even though mimics traditional news' content, lacks the traditional editorial norms that ensures the accuracy of information and appears in different forms depending on its goal and content (Iyengar and Massey 2018; Lazer et al. 2018). 


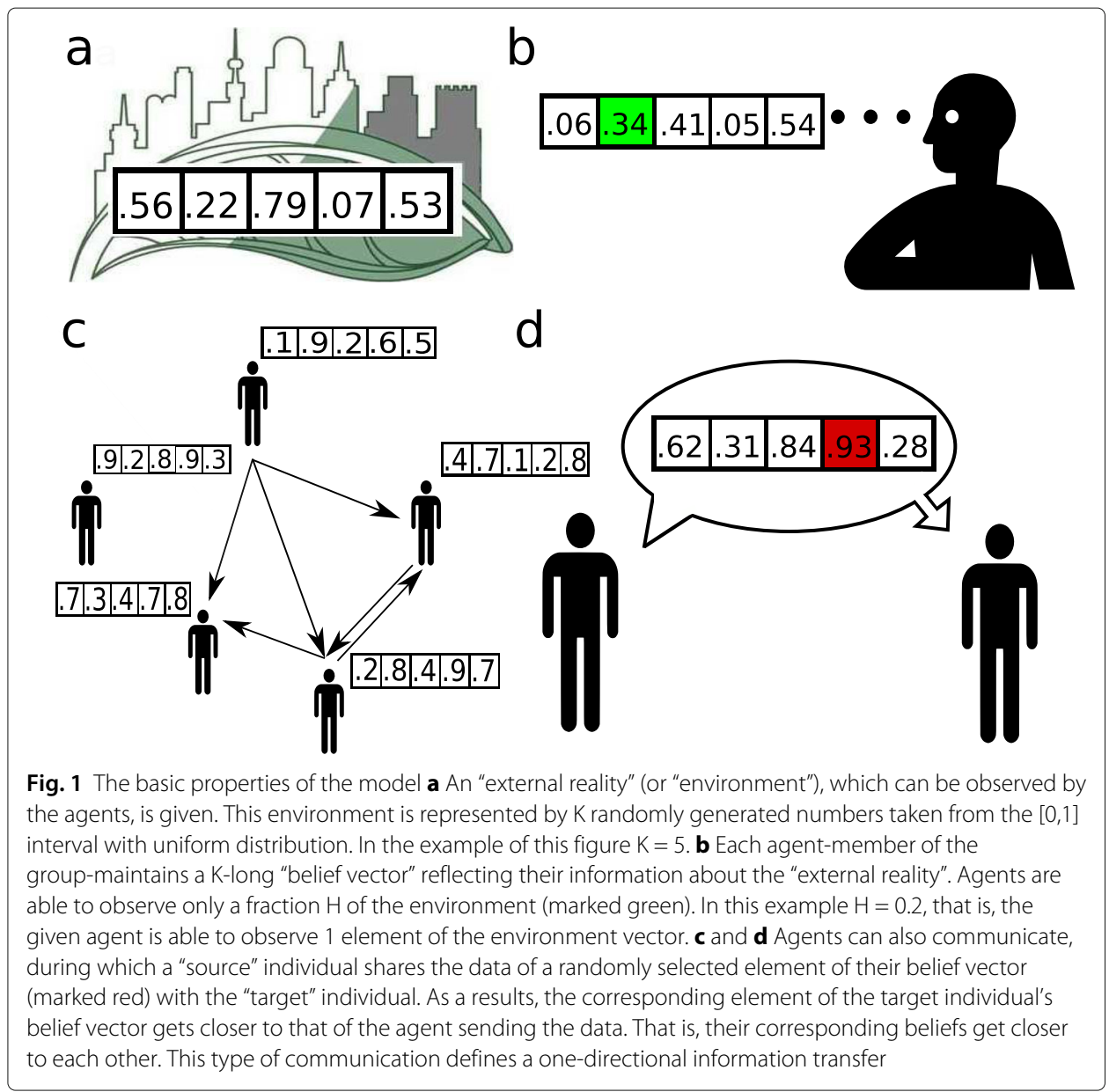

The spread of unreliable information can be traced back to various reasons: apart from the fact that people in general enjoy gossip (which often precedes news diffusion) (Szekfü and Szvetelszky 2005) they are not particularly keen on creditable information either due to psychological factors such as the confirmation bias (the preference to keep contact with people maintaining similar views, leading to the reinforcement of the original beliefs) or the desirability bias (the tendency to accept pleasing information) (Garrett and Weeks 2017; Lewandowsky et al. 2012; van Prooijen 2016) - just to mention two out of the many factors. Thus, decreasing the vulnerability of individuals to fake news, especially on social media is a major challenge, aggravated by other non-psychological factors such as the appearance of social bots or trolls who often speed up the process of spreading false information to the same order of magnitude as the propagation of reliable information (Vosoughi et al. 2018; Shao et al. 2018; Mitchell Waldrop 2017; Pennycook and Rand 2019; Bovet and Makse 2019; Varol et al. 2017).

Agent-based modelling is a popular way to study complex systems composed of many interacting autonomous units (Macal and North 2010; 2006). These models often include some kind of environment which can provide information about the spatial location of the agents or correspond to an observable external reality, similarly to our approach (Nepusz and Vicsek 2013). Regarding the belief systems of the agents, there is a whole range of representations with various levels of complexity, from very simple ones (Nepusz and Vicsek 
2013), through elaborate models (Smets and Kennes 1994; Smets 1994; Rojas-Guzmán and Kramer 2013), up to complex methods developed in the field of artificial intelligence (Bengio et al. 2013). For example, in Ref. (Chung and Reynolds 1996) the authors propose a dual inheritance evolutionary algorithm, called cultural algorithm, in which individuals maintain a shared belief system. Agent-based models tend to put an emphasis not only on the inner structure and/or behaviour of the units, but also on their interactions (Hare and Deadman 2004; Epstein and Axtell 1996; Bousquet and Le Page 2004). The models also widely differ in the goals of the agents (Vedres and Scotti 2012), such as reaching consensus (Olfati-Saber and Murray 2004) or learning to forecast in an economic environment (Bullard and Duffy 1999).

\section{The model}

Here we introduce an agent-based model in which agents are embedded into an environment that can be observed, the observation abilities of the agents are limited, and individuals can choose to modify their beliefs either by observation or communication. The "external reality" is represented by a vector of $K$ randomly selected (with a uniform distribution), independent real numbers taking values from the $[0,1]$ interval, representing $K$ independent pieces of information. We call this vector the environment. A group consists of $N$ agents, each of which maintains an image (or idea) regarding the environment. These are the belief vectors, which, along with the environment vector, are set randomly at the beginning of each run. Accordingly, the beliefs of a group at any moment can be described by $N$ real vectors, all of length $K$.

Each agent is able to "see" only a portion of the entire environment, which portion is defined by the parameter $H(H \in[0,1])$. In the present paper we focus on two cases: (i) when $H=0.1$, that is, when each agent has access only to $10 \%$ of the environment vector, and (ii) $H=1$, that is, when the agents can see the entire environment vector without any restrictions (see Fig. 1). In case $H<1$, the specific elements of the environment vector that are observable for the various agents are set randomly at the beginning of each run.

During a run, agents modify their belief vectors due to two activities: communication and/or observation. Communication is a one-directional information flow, during which the source agent $i$ shares a piece of information with the target agent $j$, who modifies their belief vector in a way that it becomes more similar to that of agent $i$ 's. For example if agent $i$ shares the 5 th element of their belief vector (corresponding to the 5th element of the environment vector) with individual $j$ then the fifth element of the belief vector of agent $j$ will get closer to that of agent $i$ 's with a random portion. That is, only the source individual influences the beliefs of the target agent. In case agent $i$ chooses to communicate, the probability of communication with agent $j$ is defined by the element $a_{i j}$ of the adjacency matrix. However, agent $i$ communicates only with probability $A_{\text {Comm }}^{i}(\leq 1)$. Accordingly, the "true" probability of communication between agents $i \rightarrow j$ is $w_{i j}=a_{i j} A_{\mathrm{Comm}}^{i}$. In the following, when we refer to the communication network, we consider this latter network, containing the $w_{i j}$ values for all the $i \rightarrow j$ agent pairs. Whether the communication makes agent $j$ better-informed or not, depends on the accuracy of the beliefs of the source agent $i$.

In contrast, observation always improves the accuracy of the beliefs of the observer. The reason why despite this fact not all agents observe the environment directly, is twofold: (i) if $H<1$ then the agents simply do not have access to all elements of the environment, 
and (ii) observation is much more costly than communication. Specifically, we assume that both activities have a cost, and that the cost of observation $C_{\mathrm{obs}}$ is much higher than the cost of communication $C_{\text {comm }}$. (Looking up the measurement data related to climate change takes much more time and energy than talking about this subject with others.)

Each run consists of several rounds during which each agent $i$ communicates and/or performs observations with probabilities $A_{\text {Comm }}^{i}$ and $A_{\text {Obs }}^{i}$, respectively.

"Fake news" is represented by a vector whose $i$ th element, $f_{i}$ is $1-e_{i}$, where $e_{i}$ is the $i$ th element of the environment vector. For example, if the sixth element of the environment vector is $e_{6}=0.37$, then the sixth element of the fake news vector will be $f_{6}=1-$ $0.37=0.63$. Considering values near 0.5 as "neutral statements" and values near 0 and 1 "extreme", this definition can be interpreted as negating the original viewpoints/pieces of information. We study three levels of exposure to fake news, $R_{\mathrm{FN}}=0 \%, 1 \%$ and $5 \%$.

Exposure to fake news is incorporated into the model as next: at the beginning of each round, a certain $\left(R_{\mathrm{FN}}\right)$ percentage of the elements of the belief vectors maintained by the group members are set to the value of the corresponding element of the fake news vector. The elements to be modified are chosen randomly.

Our question is the following: What is the optimal group structure (described by the communication network and the activities of the agents, both observation and communication) under the exposure to various levels of fake news, if by "optimal" we mean that the beliefs of the group members reflect the "reality" (represented by the environment vector)? In other words, a group is optimal, if its members can reach an accurate idea regarding their environment, despite the exposure to fake news or disinformation. We optimize the group and not the individuals, so our results reflect the interest of a group in case it aims to keep its members well-informed in the presence of disinformation.

The optimization is carried out by genetic algorithm in which the fitness function is defined as:

$$
F=\alpha_{\mathrm{Grp}}-C_{\mathrm{Actv}}
$$

where $\alpha_{\mathrm{Grp}}$ is the accuracy of the group (see Eq. 2), and $C_{\mathrm{Actv}}$ is the sum of the activity costs (Eq. 3). Since in case of uniformly distributed random values for both the initial belief vectors and the environment vector, the expected value of the initial error of the group $E_{\mathrm{GrpAvg}}^{\mathrm{Init}}$ (defined as the mean square deviation from the environment vector) is $1 / 6$, the first term of the fitness function, $\alpha_{\mathrm{Grp}}$ is:

$$
\alpha_{\mathrm{Grp}}=\frac{E_{\mathrm{GrpAvg}}^{\mathrm{Init}}-E_{\mathrm{GrpAvg}}^{\mathrm{Final}}}{E_{\mathrm{GrpAvg}}^{\text {Init }}}=\frac{\frac{1}{6}-E_{\mathrm{GrpAvg}}^{\mathrm{Final}}}{\frac{1}{6}}=1-6 E_{\mathrm{GrpAvg}}^{\mathrm{Final}}
$$

That is, the accuracy of the group is defined as the ratio of the original expected error that has been worked off during the run. (We have also run optimizations in which the initial error of the group was specified as the belief vectors' empirical mean square deviation from the environment vector, instead of the above defined expected mean square deviation. According to our results, these two approaches produce very similar outcome.) Regarding the second term of the fitness function, the activity costs, it is the average observation activity $<A_{\mathrm{Obs}}>$ multiplied by the observation cost $C_{\mathrm{obs}}$ plus the average communication activity $<A_{\text {Comm }}>$ multiplied by the communication cost $C_{\text {comm: }}$ :

$$
C_{\mathrm{Actv}}=<A_{\mathrm{Obs}}>C_{\mathrm{obs}}+<A_{\mathrm{Comm}}>C_{\mathrm{comm}}
$$




\section{Parameter settings}

In the present study we focus on small groups, counting a few dozen members, in which face-to-face communication is possible. Specifically, the parameters for the results delineated in the present paper are the following: $N=30$, where $N$ is the size of the group, and $K=20$, where $K$ is the length of the environment and fake news vectors. These parameters mainly typify families and friendship groups. Due to limitations on computational capacity, in the present paper we do not study the optimal structure of larger groups. Smaller groups have been studied ( $N=10$ and $K=10$ ) providing similar results.

Regarding the cost parameters, we have sought to satisfy the following two conditions: (i) our original assumption (namely that the cost of observation $C_{\mathrm{Obs}}$ is considerably higher than the cost of communication $C_{\text {Comm }}$ ), and (ii) staying within the boundaries of the $[0,1]$ interval, from which all elements constituting the fitness function take values. The concrete results delineated in the present paper belong to the parameter pair $C_{\mathrm{Comm}}=0.1$ and $C_{\mathrm{Obs}}=0.5$, but the conclusions hold for more extreme parameters as well satisfying our requirements (see Fig. 6b.)

The number of rounds in each run, that is, the number of rounds during which agents can communicate and/or observe their environment, is 50. The exact value of this parameter does not matter as long as it is (i) large enough for the group error to get significantly closer to its asymptotic value, but, at the same time (ii) small enough to give room for improvement originating from better group structures. Furthermore, a technical consideration is that it should not slow down the simulations unnecessarily. Once it is set, the same value is used throughout the optimization.

The detailed flowcharts of the model along with the genetic algorithm that we used for optimization are depicted in Fig. 5 in the "Methods" section.

\section{Results}

The first and most upfront observation is that exposure to fake news severely deteriorates the performance of the group. The decay is proportional to the amount of false information circulating within the group, and it holds for all values of $H$ (see Fig. 2). However, better observation abilities (corresponding to higher values of $H$ ), independently of the fake news ratio calls forth higher observation activities (Fig. 2a) resulting in better group performances, reflected by both the fitness values (Fig. 2b) and the group accuracy values (Fig. 2c). It is also clear from subfigures (b) and (c) that even high observation abilities can not compensate the exposure to false information, reflected by the constant distances among the curves.

Furthermore, according to Fig. 2a, there is a clear inverse relation between the fake news ratio and the optimal amount of communication within the group: the more a group is exposed to false information the less communication is desirable among the members, reflected by the curves marked with blue and green ' $x$ ' symbols, corresponding to $5 \%$ and $1 \%$ constant exposure rate, respectively.

Since nodes - representing group members - receive information via incoming edges and send data via outgoing edges, the weighted in-degree/out-degree properties of the nodes serve as an accurate estimate for the corresponding agent's role in the information propagation process (Albert and Barabási 2002). (The weighted in-degree of a node is the sum of the edge weights for edges in-coming to the given node, and similarly, the weighted out-degree of a node is the sum of the edge weights for edges 

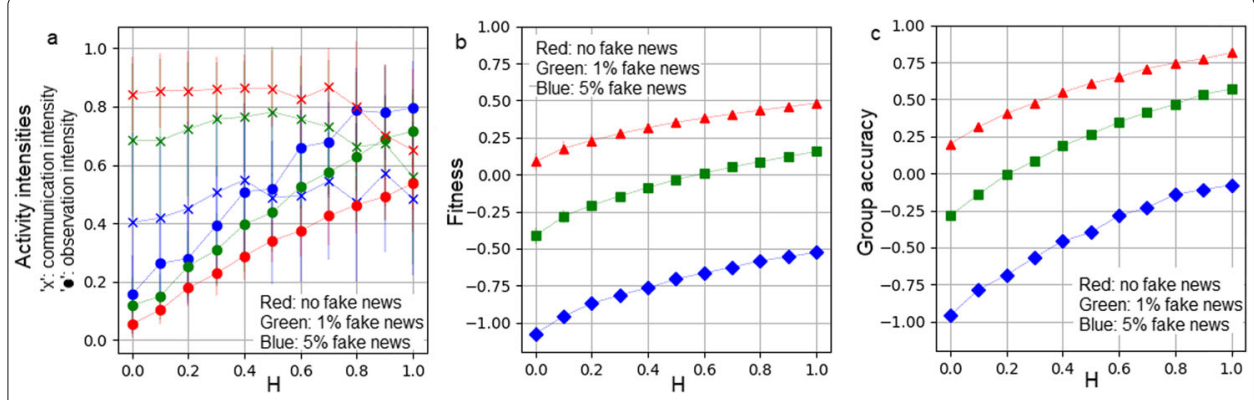

Fig. 2 a The optimal amount of activities: observation (marked with filled 'o' symbols) and communication (marked with ' $x$ ' symbols), b the fitness values, and $\mathbf{c}$ the group accuracy values, as a function of $H$, for three fake news ratios: $0 \%$ (marked with red), $1 \%$ (marked with green) and 5\%(marked with blue). a The observation activity monotonically increases as a function of $H$, meaning that members of an optimal group observe proportionally to their abilities. At the same time, at higher $\mathrm{H}$ values, the communication activities decrease, at least in case of small fake news ratio. Under exposure to fake news, there is a clear inverse relation between the fake news ratio and the optimal amount of communication. $\mathbf{b}$ and $\mathbf{c}$ As $H$ increases, the performance of the group also increases, but exposure to fake news deteriorates group-performance severely, for all $H$ values

out-going from the given node). When inspecting these properties, the first striking feature is that the weighted in-degree values are very similar for all nodes, independently of the values of $H$ or the level of false information circulating among the members (see the green bars in the insets entitled "Weighted degree distribution" in Figs. 3 and 4a). This means that all agents receive similar amount of data from their peers within an optimal group. However, the exact amount of the received data (referred to from the weighted in-degree values) does depend on the level of exposure (and also on $H$ ): along with the increment of the fake news ratio, the green bars shift towards the left, that is, towards smaller values in the insets entitled "Weighted degree distribution", and in Fig. 4a the box-plots representing higher exposure to false information (outlined with blue color) are located on significantly lower positions than the ones belonging to zero exposure ratio (outlined with red color). In other words, the amount of data flowing among members in an optimal group is inversely related to the group's exposure to fake news. (Note that this feature is in agreement with the decreased communication activities, shown in Fig. 2a).

In contrast, the out-degrees - reflecting the participation in spreading the information - behave very different: in case $H=0.1$, that is, when each agents have access only to a small portion of the environment (10\%, in this case) and there are no false information circulating, the best strategy to stay well-informed is to maintain a basically full graph in which everybody is connected to everybody else in both directions (receiving and sending data). This property is reflected by the uniformly high in-degree and out-degree values in the histogram entitled "Weighted degree distribution" in Fig. 3a. However, as the ratio of the fake news increases (bottom row), the out-degree values start to disperse, marking a differentiation of the members regarding their activity in spreading the information. Specifically, a significant portion of the members cease to participate in circulating data, and remain connected to the group only via their in-coming edges (peripheral nodes on the graphs in Fig. 3c and d) while others remain active.

Surprisingly, similar differentiation occurs with the increment of $H$ (more specifically, when $H=1$ ) in case there is no exposure to fake news. In other words, in an optimal group, in the ideal case when there is no false information circulating among the 


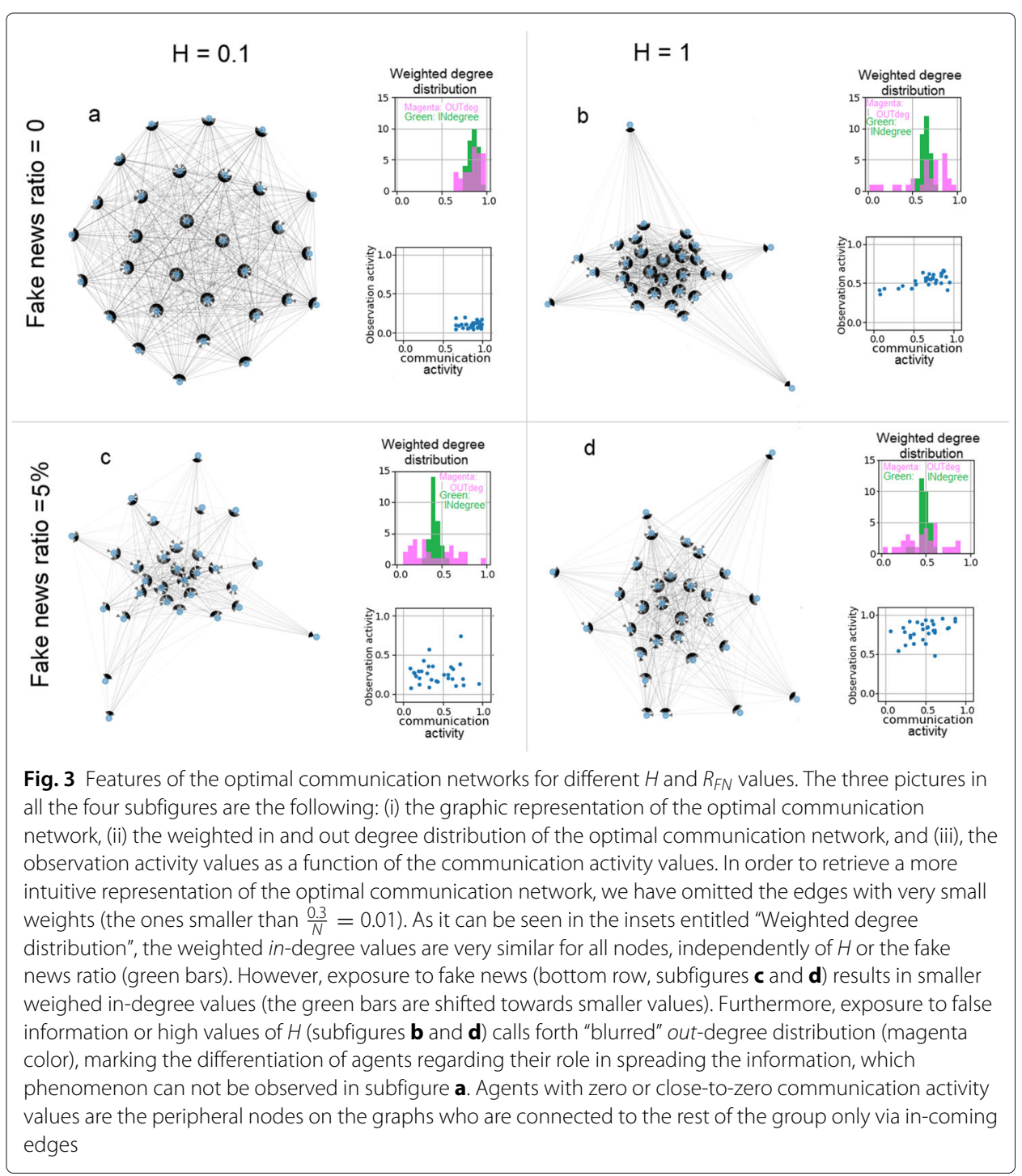

members, agents specialize with regard to their information spreading activity in case all agents have full access to the entire environment $(H=1)$, and maintain a full network in which everybody is connected equally to everybody else in case the access to information is limited ( $H=0.1$ ) (see the top row of Fig. 3). Although diversity has been reported to be advantageous from many points of views (Page 2010), the above phenomenon is still remarkable, since here we have a case in which originally similar agents specialize themselves with respect to their function within the group, under certain conditions.

According to the insets depicting the observation activities as a function of the communication activities (bottom insets in Fig. 3), peripheral agents (maintaining small communication activities) are not characterized by higher observation activities, rather especially in subfigure (b) - the observation and communication activities correlate with each other (agents with smaller communication activities have smaller observation activities as well). This correlation relaxes in case of exposure to fake news marking the appearance of agents with diverse characteristics. 

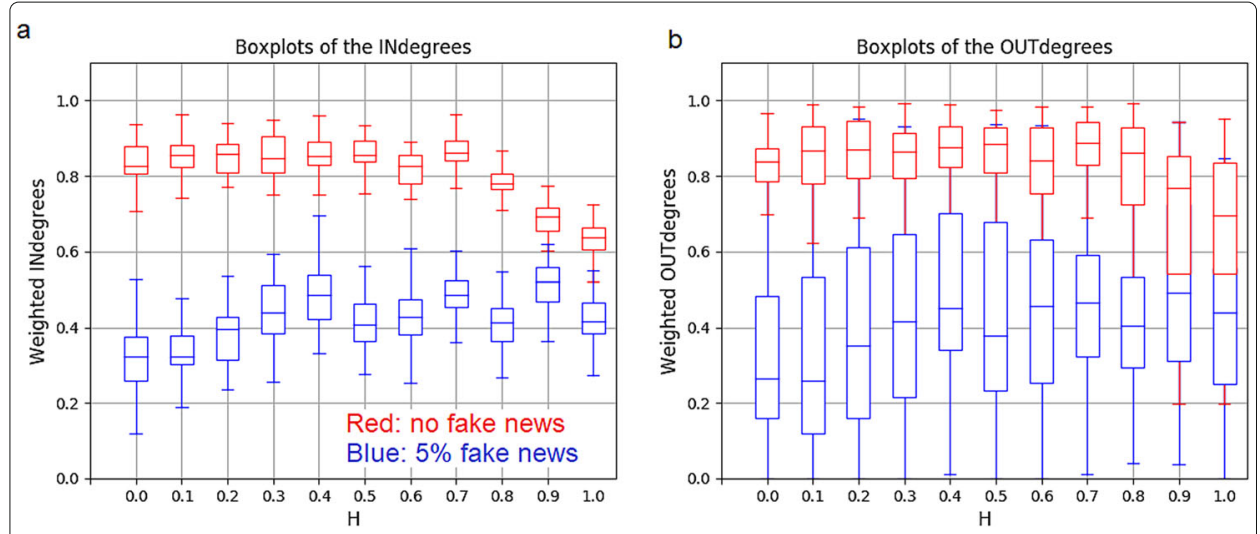

Fig. 4 Box-plots of the $\mathbf{a}$ in-degrees and $\mathbf{b}$ out-degrees of the nodes of the optimized communication networks as a function of $\mathrm{H}$, for $5 \%$ and $0 \%$ fake news ratios. In both subfigures blue represents $5 \%$ fake news ratio, and red denotes the case when there are no fake news. The weighted in-degree values of the agents are much more homogeneous than their weighted out-degree values independently of $H$ or $R_{F N}$. As a general rule, exposure to fake news calls forth smaller in-degree and out-degree values, in agreement with the decreased communication activity. Exposure to fake news also disperses the out-degree values, marking the diversification of agents regarding their information-spreading abilities

\section{The scope of the model and main results}

In the present study we assume (i) small groups counting a few dozen members in which face-to-face communication is possible, (ii) that communication is information flow during which an agent modifies the beliefs of an other agent, (iii) equal information accessing abilities, (iv) the existence of an "external reality" that can be observed, and (v) in some cases, the presence of fake news, meanwhile we omit psychological factors such as confirmation bias or desirability bias. In real life, these assumptions hold for small communities, such as families or smaller circles of friends aiming to be wellinformed regarding some observable data within their environment. We show that in case of exposure to fake news, specialization with respect to information spreading activity among the members is beneficial, along with intensified observation and weakened communication activity. Furthermore, observation activity - independently of the level of fake news - should increase as a function of $H$, that is, better access to information in general should give rise to more observation. Regarding the communication activity, higher exposure to fake news makes intense communication counterproductive, even in the extreme case when $C_{C o m m}=0$, that is, when there is no cost associated with communication (see Fig. 6b).

\section{Methods}

The code was written in Python. In order to find the optimal communication network and activity levels, we have optimized these values by using a genetic algorithm (Eiben and Smith 2010). Figure 5a depicts the flowchart of the used algorithm whose input are the parameters and output is a population of optimized groups, each represented by a so called "chromosome". In the corresponding literature, "chromosome" refers to an instance of those parameters that the algorithm aims to optimize. Accordingly, in the present model, each chromosome contains: 


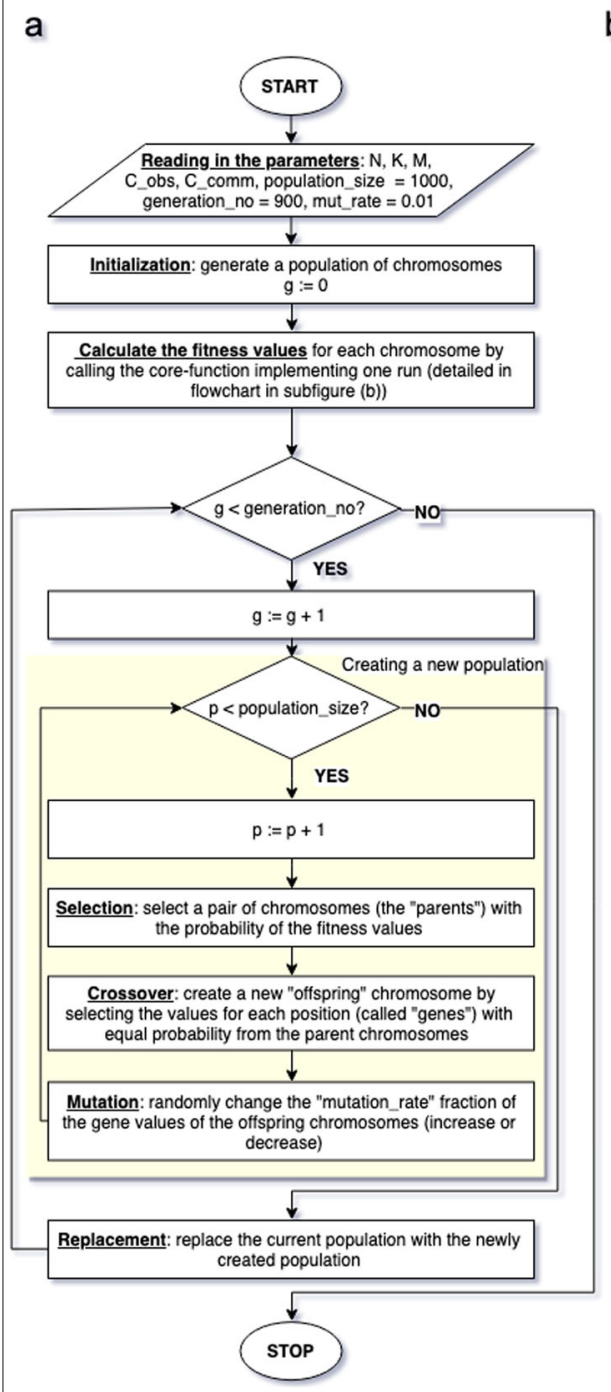

b

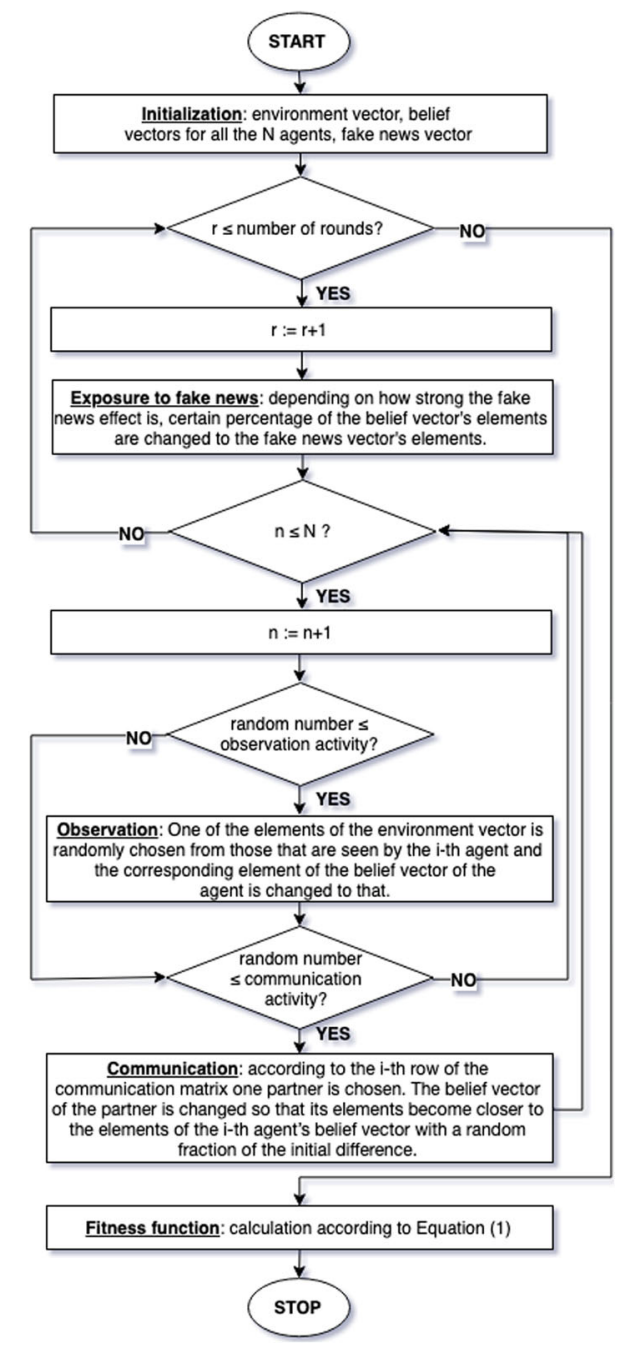

Fig. 5 The flowchart of the $\mathbf{a}$ genetic algorithm and $\mathbf{b}$ the routine assigning a fitness value for each chromosome. a Genetic algorithms - a popular and widely applied optimization approach - are designed to optimise a population of chromosomes with respect to a pre-defined fitness function. $\mathbf{b}$ A crucial part of all genetic algorithms is the way a fitness value is assigned to a chromosome: the input of this function is an instance of chromosome ( $A$ and $B$ matrices) and the output is the corresponding fitness value measuring the "quality" of the input. The first two decision boxes (diamond shapes) stand for two for-loops, while the second two decision boxes stand for two if-statements

- An adjacency matrix $A:=\left(a_{i, j}\right)_{N x N}$ whose values are taken from the [0,1] closed interval. In this matrix, element $\left(a_{i, j}\right)$ defines the probability of communication between agents $i \rightarrow j$, in case agent $i$ chooses to communicate in a certain round. Accordingly - since these values define probabilities - the sum of each row is always normalized to 1 , and the elements in the main diagonal are set to 0 (since agents do not communicate with themselves).

- A vector $A_{\text {Comm }}:=\left(A_{\text {Comm }}^{i}\right)_{N}$ whose $i$ th element $A_{\text {Comm }}^{i}$ is the communication activity of agent $i$, and

- A vector $A_{\mathrm{Obs}}:=\left(A_{\mathrm{Obs}}^{i}\right)_{N}$ whose $i$ th element $A_{\mathrm{Obs}}^{i}$ is the observation activity of agent $i$. 
The elements of the vectors $A_{\mathrm{Comm}}$ and $A_{\mathrm{Obs}}$ are taken from the $[0,1]$ closed interval as well.

At the beginning of the optimization process, in the "Initialization step" (second box in Fig. 5a) the values of the chromosome, that is, the elements of the matrix $A$ and the vectors $A_{\text {Comm }}$ and $A_{\mathrm{Obs}}$ are taken from the $[0,1]$ closed interval with uniform random distribution.

Throughout the optimization process these values become more and more "optimal" with respect to a so called fitness function - this is what a genetic algorithm is designed for (Eiben and Smith 2010). This fitness function returns a fitness value for each chromosome reflecting its quality, that is, it is a numeric measure designed to describe how well a certain chromosome solves the original problem. Figure $5 \mathrm{~b}$ shows the flowchart of the function we have used to assign a fitness value for each chromosome: its input is (i) a communication network and (ii) the activity values for all agents (both observation and communication), and its output is a fitness value. The exact formula of the fitness function is defined by Eq. 1

Regarding the $A_{i, j}$ adjacency matrix, it is important to highlight that its element $a_{i j}$ refers to the probability of communication between agents $i \rightarrow j$, in case agent $i$ chooses to communicate in a certain round. However - as emphasized in "The model" section as well - agent $i$ does not necessary communicate in each round, but only in a portion of them, defined by the $A_{\text {Comm }}^{i}(\leq 1)$ communication activity. Hence, the real ("effectuating") probability of communication between members $i \rightarrow j$ is $w_{i j}=a_{i j} A_{\text {Comm }}^{i}$. The results reported in the manuscript refer to this latter "effective" communication network, defined by the $w_{i j}$ values. Since there is a one-to-one mapping between graphs and matrices, the above adjacency matrix along with the communication activities unequivocally defines the communication network (Bollobás 2002).

As seen in Fig. 5a, the genetic algorithm itself also has parameters which are independent from the original problem. Namely, the attribute population_size sets the number of chromosomes in each generation, while the parameter generation_no defines the number of generations during the entire optimization process. The first parameter, population_size, is the analogous of the genetic diversity within a (biological) population. In case of optimization problems, however, its proper size depends on two (contradicting) considerations: on the one hand, larger population sizes result more diverse "solution-propositions" in each generations rendering the appearance of better and better solutions probable, but, on the other hand, too large population sizes entail slower convergences, manifesting themselves in unnecessarily long computation times (unnecessarily high number of generations). Thus, the optimal value for population_size is defined by the balance of the above two aspects (Gotshall and Rylander 2002; Alander 1992; Roeva et al. 2013). Although it follows from the foregoing that the parameter generation_no is related to the parameter population_size, its proper value can be defined based on the shape of the so called "fitness curve" which is the curve depicting the average fitness values as a function of the generation number. At the beginning of the optimization process (at low generation numbers) the increase is fast, which, after a while slows down and finally vanishes: this is when the fitness curve "saturates" indicating that the chromosomes in the last generations are optimal solutions for the original problem (see Fig. 6a). Keeping these considerations in mind, we have set 

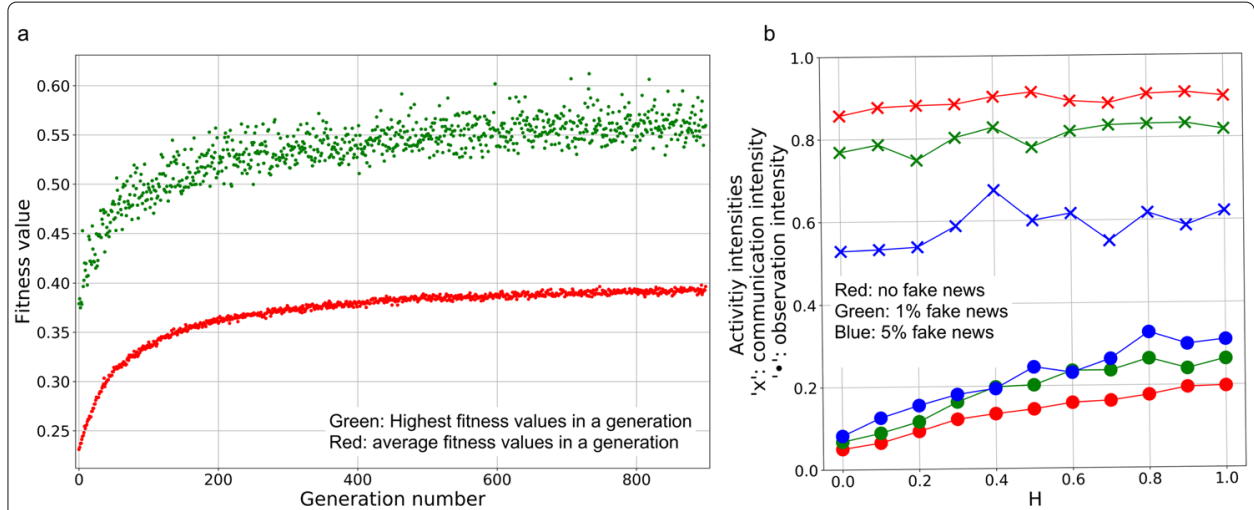

Fig. 6 The progress of the optimization (a) and the optimal activity values for extreme cost values (b). a The best (green dots) and the average (red dots) fitness values as a function of the generation number. Saturating fitness curve indicates that the chromosomes in the last generations are optimal solutions for the original problem. $\mathbf{b}$ Optimal activity values for as a function of $H$ for $C_{C o m m}=0$ and $C_{O b s}=1$. According to our results, exposure to fake news calls forth intensified observation and weakened communication activity. Observation activities in optimized groups - independently of the level of fake news - increase as a function of $H$. In contrast to the observation activities (marked by filled circles), higher exposure to fake news makes intense communication counterproductive, marked by the blue ' $x$ ' marks which stay around 0.6 , despite the fact that no cost is associated to it

the parameters population_size $=1000$ and generation_no $=900$. The chromosomes in the last generations converge, that is, they are very similar to each other. Due to this effect, it is reasonable to average them and define the solution as the average of the population_size $(=1000)$ chromosomes of the last generation. The reported results are obtained in this way.

\section{Acknowledgements}

Not applicable.

\section{Authors' contributions}

$A Z$ and ID designed the model and analyzed the results. AZ and EB made the code and run the optimization. AZ was a major contributor in writing the manuscript to which EB contributed with the literature overview in the Introduction. All authors read and approved the final manuscript.

\section{Funding}

The research was partially supported by the European Union through projects 'RED-Alert' (grant no: 740688-RED-Alert-H2020- SEC-2016-2017/H2020- SEC-2016-2017-1) and by the Hungarian National Research, Development and Innovation Office (grant no. K 128780). We acknowledge further partial support by the Bolyai János Research Scholarship, the Bolyai+ Research Scholarship (grant no. ÚNKP-18-4) founded by the New National Excellence Program of the Ministry of Human Capacities and the National Research, Development and Innovation Office.

\section{Availability of data and materials}

All data generated and analyzed during this study are included in this article. (See the "Parameter settings" subsection within the "The model" section, and the "Methods" section.)

\section{Competing interests}

The authors declare that they have no competing interests.

Received: 16 May 2019 Accepted: 22 October 2019

Published online: 08 November 2019

\section{References}

Alander JT (1992) On optimal population size of genetic algorithms. In: CompEuro 1992 Proceedings Computer Systems and Software Engineering. pp 65-70. https://doi.org/10.1109/CMPEUR.1992.218485

Albert R, Barabási A-L (2002) Statistical mechanics of complex networks. Rev Mod Phys 74:47-97. https://doi.org/10.1103/ RevModPhys.74.47

Bengio Y, Courville A, Vincent P (2013) Representation learning: A review and new perspectives. IEEE Trans Pattern Anal Mach Intell 35:1798-1828. https://doi.org/10.1109/TPAMI.2013.50

Berger PL, Luckmann T (1991) The Social Construction of Reality: A Treatise in the Sociology of Knowledge. Penguin Press, London 
Bollobás B (2002) Modern Graph Theory. Springer, New York

Bousquet F, Le Page C (2004) Multi-agent simulations and ecosystem management: A review. Ecol Model 176:313-332. https://doi.org/10.1016/j.ecolmodel.2004.01.011

Bovet A, Makse AH (2019) Influence of fake news in twitter during the 2016 us presidential election. Nat Commun 10. https://doi.org/10.1038/s41467-018-07761-2

Bullard J, Duffy J (1999) Using genetic algorithms to model the evolution of heterogeneous beliefs. Comput Econ 13:41-60. https://doi.org/10.1023/A:1008610307810

Chung CC-J, Reynolds RG (1996) A testbed for solving optimization problems using cultural algorithms. Evol Program. Published in Evolutionary Programming 1996. http://citeseerx.ist.psu.edu/viewdoc/summary?doi=10.1.1.55.3116

Eiben AE, Smith JE (2010) Introduction to Evolutionary Computing. Springer, Heidelberg

Epstein J, Axtell R (1996) Growing Artificial Societies: Social Science from the Bottom Up, Vol. 76. https://doi.org/10.2307/ 20048043

Garrett RK, Weeks B (2017) Epistemic beliefs' role in promoting misperceptions and conspiracist ideation. PLoS ONE 12:0184733. https://doi.org/10.1371/journal.pone.0184733

Gotshall SP, Rylander B (2002) Optimal population size and the genetic algorithm. https://www.semanticscholar.org/ paper/Optimal-Population-Size-and-the-Genetic-Algorithm-Gotshall-Rylander/ 6eb8128b679348b5bef2729c56dd31f0f173a60b

Hacking I (2000) The Social Construction of What?. Harvard University Press, Cambridge

Hare M, Deadman P (2004) Further towards a taxonomy of agent-based simulation models in environmental management. Math Comput Simul 64:25-40. https://doi.org/10.1016/S0378-4754(03)00118-6

lyengar S, Massey D (2018) Scientific communication in a post-truth society. Proc Natl Acad Sci 116:201805868. https:// doi.org/10.1073/pnas.1805868115

Lazer MJD, Baum M, Benkler Y, Berinsky JA, Greenhill MK, Menczer F, Metzger JM, Nyhan B, Pennycook G, Rothschild D, Schudson M, Sloman S, Sunstein C, Thorson AE, Watts JD, Zittrain LJ (2018) The science of fake news. Science 359:1094-1096. https://doi.org/10.1126/science.aao2998

Lewandowsky S, Ecker U, Seifert C, Schwarz N, Cook J (2012) Misinformation and its correction continued influence and successful debiasing. Psychol Sci Pub Interes 13:106-131. https://doi.org/10.1177/1529100612451018

Macal C, North M (2006) Tutorial on agent-based modeling and simulation part 2: How to model with agents. pp 73-83. https://doi.org/10.1109/WSC.2006.323040

Macal C, North M (2010) Tutorial on agent-based modelling and simulation. J Simul 4:151-162. https://doi.org/10.1057/ jos.2010.3

Mitchell Waldrop M (2017) News feature: The genuine problem of fake news. Proc Natl Acad Sci 114:201719005. https:// doi.org/10.1073/pnas.1719005114

Nepusz T, Vicsek T (2013) Hierarchical self-organization of non-cooperating individuals. PloS ONE 8:81449. https://doi.org/ 10.1371/journal.pone.0081449

O'Connor C, Weatherall JO (2018) The Misinformation Age: How False Beliefs Spread. Yale University Press, New Haven

Olfati-Saber R, Murray R (2004) Consensus problems in networks of agents with switching topology and time-delays. IEEE Trans Autom Control 49. https://doi.org/10.1109/tac.2004.834113

Page SE (2010) Diversity and Complexity. Princeton University Press, Princeton

Pennycook G, Rand D (2019) Fighting misinformation on social media using crowdsourced judgments of news source quality. Proc Natl Acad Sci 116:201806781. https://doi.org/10.1073/pnas.1806781116

Roeva O, Fidanova S, Paprzycki M (2013) Influence of the population size on the genetic algorithm performance in case of cultivation process modelling. In: Proceedings of the 2013 Federated Conference on Computer Science and Information Systems. pp 371-376. https://annals-csis.org/Volume_1/pliks/167.pdf

Rojas-Guzmán C, Kramer MA (2013) GALGO: A genetic algorithm decision support tool for complex uncertain systems modeled with bayesian belief networks. CoRR abs/1303.1498. 1303.1498

Searle JR (2011) Making The Social World. Oxford University Press, New York

Scheufele DA, Krause NM (2019) Science audiences, misinformation, and fake news. Proc Natl Acad Sci 116(16):7662-7669. https://doi.org/10.1073/pnas.1805871115. https://www.pnas.org/content/1 16/16/7662.full.pdf

Shao C, Luca Ciampaglia G, Varol O, Flammini A, Menczer F, Yang K-C (2018) The spread of low-credibility content by social bots. Nat Commun 9. https://doi.org/10.1038/s41467-018-06930-7

Sloman S, Fernbach P (2017) The Knowledge Illusion: The Myth of Individual Thought and the Power of Collective Wisdom. Macmillan; Main Market edition, New York

Smets P (1994) The transferable belief model for quantified belief representation 1. https://doi.org/10.1007/978-94-0171735-9

Smets P, Kennes R (1994) The transferable belief model. Artif Intell 66:191-234. https://doi.org/10.1016/00043702(94)90026-4

Szekfü B, Szvetelszky Z (2005) Three degrees of inclusion: the gossip-effect in human networks. AlP Conf Proc. https://doi. org/10.1063/1.1985397

van Prooijen JW (2016) Why education predicts decreased belief in conspiracy theories: Education and conspiracy beliefs. Appl Cogn Psychol 31. https://doi.org/10.1002/acp.3301

Varol O, Ferrara E, Davis C, Menczer F, Flammini A (2017) Online human-bot interactions: Detection, estimation, and characterization. In: Proceedings of the Eleventh International AAAI Conference on Web and Social Media. pp 280-289

(2012) Networks in Social Policy Problems(Vedres B, Scotti M, eds.). Cambridge University Press, Cambridge UK

Vosoughi S, Roy D, Aral S (2018) The spread of true and false news online. Science 359:1146-1151. https://doi.org/10.

1126/science.aap9559

\section{Publisher's Note}

Springer Nature remains neutral with regard to jurisdictional claims in published maps and institutional affiliations. 